\title{
EDITORIALS
}

\section{Patient Aligned Care Teams (PACT): VA's Journey to Implement Patient-Centered Medical Homes}

\author{
Elizabeth M. Yano, PhD, MSPH ${ }^{1,2}$, Matthew J. Bair, MD, MS 3,4 , Olveen Carrasquillo, MD, MPH \\ Sarah L. Krein, PhD, $R N^{6,7}$, and Lisa V. Rubenstein, MD, MSPH $1,2,8,9,10$
}

'VA HSR\&D Center for the Study of Healthcare Innovation, Implementation and Policy, VA Greater Los Angeles Healthcare System, Sepulveda, CA, USA; 'Department of Health Policy and Management, UCLA Fielding School of Public Health, Los Angeles, CA, USA; ${ }^{3}$ Roudebush VA Medical Center, VA Center for Health Information and Communication, Indianapolis, IN, USA; ${ }^{4}$ Indiana University School of Medicine, Indianapolis, IN, USA; 5 Miller School of Medicine, University of Miami, Miami, FL, USA; ${ }^{6}$ VA Ann Arbor Health Care System, Ann Arbor, MI, USA; ${ }^{7}$ University of Michigan Medical School, Ann Arbor, MI, USA; ${ }^{8}$ VA QUERI Center for Implementation Practice and Research Support, VA Greater Los Angeles Healthcare System, Sepulveda, CA, USA; ${ }^{\circ}$ Department of Medicine, VA Greater Los Angeles Healthcare System and UCLA Geffen School of Medicine, Los Angeles, CA, USA; ${ }^{10}$ RAND Health, RAND Corporation, Santa Monica, CA, USA.

J Gen Intern Med 29(Suppl 2):S547-9

DOI: $10.1007 / \mathrm{s} 11606-014-2835-8$

(c) Society of General Internal Medicine 2014

$\mathrm{I}$ n 2010, the US Department of Veterans Affairs (VA) launched national implementation of patient-centered medical homes (PCMH) through the Patient Aligned Care Teams (PACT) initiative, as described by Gordon Schectman and Richard Stark, the chief architects of PACT, in their commentary to this Supplement. Concurrently, the PACT initiative aimed to incorporate rigorous formative and summative evaluation of PACT implementation and to promote PACTrelated innovation development and testing. To accomplish these goals, the initiative established a national evaluation team (based in Seattle) and competitively funded five PACT Demonstration Laboratories (in Ann Arbor, Los Angeles, Iowa City, Philadelphia, and Portland). In addition, embedded health services researchers and clinical leader partners from outside the Demonstration Laboratories undertook relevant studies. Together, the implementation and evaluation components of PACT as reported in this Supplement's articles document opportunities and challenges in implementing a PCMH in integrated healthcare systems that are both specifically relevant to VA and informative to other managed care or Accountable Care Organizations (ACOs) engaged in implementing PCMH models. In addition, as highlighted in the Reid and Wagner commentary in this Supplement, the articles have implementation science implications as they delve into components of the Chronic Illness Care Model.

Rather than a demonstration project, VA's implementation of PACT represents a national rollout of PCMH to all VA primary care practices in more than 150 medical centers and over 800 community-based outpatient clinics. The articles in this Supplement therefore reflect realistic transformation on a national scale during its first 2 to 3 years.

Published online April 9, 2014
Funded by the VA Office of Patient Care Services' Primary Care Program Office, this Journal of General Internal Medicine (JGIM) Supplement shares the lessons learned by researchers and their clinical and policy partners during early stages of PACT implementation. The 19 published articles underwent rigorous JGIM peer review along with nearly 50 additional manuscripts that were submitted in response to the supplement's Call for Papers but not selected for the Supplement. The published articles touch upon virtually all facets of medical homes, including implementation strategies, performance measurement, care transitions, team development, mental health and pharmacy integration, quality improvement, and medical home neighborhood development.

We organized the PACT Supplement to reflect different stages and levels of medical home implementation, starting with pre-implementation PACT challenges and insights about implementation strategies, closer examination of organizational structures, strengths and challenges of implementing team-based care, and ending with implications for specialty care relationships and addressing the needs of special populations.

\section{PRIMARY CARE CHALLENGES PRIOR TO PACT IMPLEMENTATION}

Farmer and colleagues surveyed all frontline primary care directors to better understand their top management challenges prior to PACT implementation. They found 23 to $80 \%$ of all VA practices reported moderate-to-extreme challenges in virtually all PCMH domains and features queried in the survey. The greatest challenges related to clinical informatics (e.g., clinical reminder volume, medical record alerts, and time to document notes) and limited technical support. Other major challenges included managing primary care patients' chronic pain, 
mental illness, and substance abuse, as well as problems coordination with non-VA care.

\section{IMPLEMENTATION STRATEGIES AND EXPERIENCES}

PACT has required intensive national, regional, and local implementation efforts. Among these, Bidassie and colleagues describe VA's experience disseminating a national Breakthrough Series Collaborative method, involving 141 teams spanning over 1,500 Plan-Do-Study-Act (PDSA) quality improvement (QI) projects. An evaluation of a national online repository of ready-to-use tools created to facilitate PACT implementation is described by Luck and colleagues. Impressively, more than 6,000 staff used the repository in the first 19 months of its availability.

PACT Demonstration Laboratories also describe regional QI initiatives. Butler's virtual QI Collaborative supported regional PACT implementation and spread without the need for in-person meetings, while Rubenstein's evidence-based quality improvement approach tested new infrastructure and methods for promoting ongoing primary care improvement and innovation in PACT. At the facility level, Chang et al. applied a locally tailored continuous QI process to understand communication barriers between PACT and mental health providers and initiate a change strategy. The strategy used has implications for improving medical home coordination with other types of specialists.

Eliciting general staff experiences during PACT implementation revealed important insights. Kansagara and colleagues report staff perceptions of PACT performance metrics and potential unintended consequences of metric reporting. They provide evidence that staff perceived some metrics as misaligned with PACT principles, team-based care, or patient priorities. Tuepker describes early enthusiasm among primary care staff for PACT concepts juxtaposed with staff concerns. These included inadequate implementation resources, limited staffing, lack of dedicated training opportunities, and time constraints that interfered with team development.

\section{PACT TEAM-BASED CARE}

Several articles focused explicitly on PACT team-based care, emphasizing the teamlets (provider, nurses, medical assistants, clerks) that are at the heart of PACT's redesign of primary care staffing. Rodriguez and colleagues found that teamlet members reported receiving little input to guide their development and limited training at the teamlet level. They also report chronic under-staffing and describe territorial attitudes when staff had to cover other areas. True et al. found that effective teamwork and task delegation required well-demarcated boundaries, a sense of collective identity, shared goals, and a sense of purpose. Forman and colleagues echo Rodriguez's findings about the need for sufficient staffing, demonstrating that the issue is not isolated to one facility or region. They also highlight problems related to teamlet members who are not colocated and communication challenges with trainees or part-time providers. Their assessment was mirrored in Wang's study of team-based solutions to disruptions caused by end-of-medicine-residency transitions. Most PACT members (and residents) agreed that a more formal approach to transitions was needed, including formal handoffs to non-physician teamlet members.

Helfrich's paper builds on these local and regional findings by reporting worrisome results from a national survey of over 4,500 primary care providers and staff. Two years into PACT implementation, they found almost $40 \%$ of respondents reported burnout. Burnout was associated with perceived gaps in participatory decision-making, insufficiently staffed PACT teams, time spent on tasks someone with less training could do, and a stressful, fast-moving work environment.

To further incentivize and guide PACT implementation, the PACT initiative added several additional performancerelated metrics (e.g., the PACT Compass) to the VA's already existing extensive audit-and-feedback performance monitoring system. Yet, in interviews spanning 16 VA medical centers, Hysong and colleagues highlighted the challenges of improving performance assessment when measures are attributed largely to individual providers rather than teams.

PACT role changes also have implications for effective and efficient teamwork. For example, O'Neill and colleagues report on a comparison of primary care physician vs. clinical pharmacist directed nurse case management of PACT patients with poorly controlled hypertension. Their findings suggest that clinical pharmacists can supervise multiple nurse care managers in titrating medications for patients with poorly controlled hypertension, thus potentially reducing bottlenecks related to physician access by the nurses and increasing nurse care management capacity.

PACT aimed, as core objectives, to improve both continuity with individual providers and access to them. Katz and colleagues examined the relationships between continuity - a high-value PACT attribute - and patient-provider communication. In this study, decreased primary care provider continuity was associated with significantly lower perceived quality of patient-provider communication. Werner and colleagues, in turn, examined the relationship between access and emergency room (ER) use. Using national VA data, they found that the absence of a primary care provider for a week or more had a small but statistically significant relationship to increased ER visits, especially if that provider was the sole or nearsole provider of a patient's care. Combined, these findings support the importance of both continuity and 
access as well as the need to reduce current variations in these key PACT features.

\section{PACT FOR SPECIAL POPULATIONS}

While early PACT implementation focused on primary care-based organizational changes, two articles in this supplement remind us of the importance of considering primary care received outside of general primary care settings. Fix and colleagues describe wide variations in delivery of PACT-principled care in VA HIV specialty care clinics and found that patients were less satisfied with care in the HIV clinics that had features that were less PACT-aligned. Yano and colleagues similarly raise implications for PACT around delivering gender-sensitive comprehensive care to female veterans, who may be seen in separate women's health clinics or in general primary care clinics with varying levels of readiness for handling women's primary care needs.

In summary, we consider this set of articles examining early stages of medical home implementation in the VA healthcare system to be a valuable resource for clinician leaders, policy makers, and primary care researchers. We are excited and pleased to present them in this JGIM supplement. 